\title{
Estrategias de afrontamiento para el manejo del estrés y su relación con el nivel de obesidad y calidad de vida en pacientes candidatos a cirugía bariátrica
}

\author{
María Eugenia Terrazas-Romero, ${ }^{1}$ Guadalupe Jazmín Flores-Boyso, ${ }^{1}$ Nallely Teotetl Zenil-Magaña, ${ }^{1}$ \\ Edgar Landa-Ramírez ${ }^{1}$ y Aída Monserrat Reséndiz-Barragán ${ }^{2}$ \\ ${ }^{1}$ Universidad Nacional Autónoma de México, Facultad de Psicología, Programa de Psicología de la Salud y Medicina Conductual, Ciudad de México, \\ México; ${ }^{2}$ Secretaría de Salud, Hospital General "Dr. Manuel Gea González", Subdirección de investigación Biomédica, Ciudad de México, México
}

\section{Resumen}

Introducción: El estrés es una variable que puede desempeñar un papel importante en el desarrollo de la obesidad y en la calidad de vida de los pacientes candidatos a cirugía bariátrica, ya que se vincula a la ingesta de alimentos hipercalóricos, sedentarismo y mayor incidencia de trastornos psicológicos. Objetivo: Describir el tipo y estrategias de afrontamiento al estrés más frecuentes en los pacientes candidatos a cirugía bariátrica y establecer su relación con el índice de masa corporal y su percepción de calidad de vida. Método: Se evaluaron 101 pacientes con obesidad severa candidatos a cirugía bariátrica por medio del Cuestionario de Afrontamiento al Estrés y el Inventario de Calidad de Vida y Salud. Resultados: El tipo de afrontamiento más utilizado es la acción y la estrategia más frecuente es la focalización en solución de problemas. Cinco estrategias de afrontamiento se asociaron significativamente con la calidad de vida; no hubo asociación significativa con el índice de masa corporal. Conclusión: Existe asociación entre algunos estilos de afrontamiento y la calidad de vida de los individuos candidatos a cirugía bariátrica.

PALABRAS CLAVE: Obesidad. Cirugía bariátrica. Estrés psicológico. Calidad de vida.

\begin{abstract}
Introduction: Stress is a variable that may play an important role in the development of obesity and in the quality of life of patients who are candidates for bariatric surgery, since stress is linked to the consumption of hypercaloric foods, sedentariness and higher incidence of psychological disorders. Objective: To describe the most common types of stress coping strategies in patients who are candidates for bariatric surgery and establish their relationship with body mass index and quality of life perception. Method: One-hundred and one patients with severe obesity who were candidates for bariatric surgery were evaluated by means of the Stress Coping Questionnaire and the Quality of Life and Health Inventory. Results: The most commonly used coping type is action and the most common strategy is focus on problem solving. Five coping strategies were significantly associated with quality of life; there was no significant association with body mass index. Conclusion: There is an association between some coping styles and quality of life in individuals who are candidates for bariatric surgery.
\end{abstract}

KEY WORDS: Obesity. Bariatric surgery. Psychological stress. Quality of life.

Correspondencia:

Aída Monserrat Reséndiz-Barragán

E-mail: aidaresendiz@ hotmail.com
Fecha de recepción: 30-01-2018

Fecha de aceptación: 18-07-2018

DOI: 10.24875/GMM.18004175
Gac Med Mex. 2018;154:569-574

Disponible en PubMed

www.gacetamedicademexico.com 


\section{Introducción}

El incremento global de la obesidad se ha hecho evidente al igual que la preocupación por determinar las causas de dicho fenómeno. Las cifras de la Organización Mundial de la Salud indican que mil millones de personas en todo el mundo tienen un índice de masa corporal (IMC) indicativo de sobrepeso (IMC de 25) y al menos 300 millones padecen obesidad (IMC de 30), lo cual constituye un factor de riesgo para desarrollar diversos problemas de salud, entre ellos diabetes e hipertensión. ${ }^{1-3}$ De acuerdo con la Organización para la Cooperación y Desarrollo Económico, Estados Unidos y México presentan la mayor prevalencia en obesidad en el mundo; $30 \%$ de la población en México (34.5\% de las mujeres adultas y $24.2 \%$ de los hombres), lo cual equivale a 36 millones de personas. ${ }^{4}$ Como consecuencia, en México se han incrementado los casos de diabetes tipo 2, dislipidemias e hipertensión y se calcula que en los años venideros el aumento en la carga de padecimientos al sistema de salud prácticamente se duplicará. ${ }^{4,5}$

Se han identificado diversos factores genéticos, psicológicos y sociales involucrados en la etiología de la obesidad y que afectan la calidad de vida, entre ellos al estrés psicosocial. ${ }^{6,7}$ Se ha reportado que este puede llevar a las personas a elegir alimentos altos en grasa y con alta densidad energética, así como a que muestren menor disponibilidad para preparar alimentos saludables y realizar actividades físicas y recreativas, lo cual puede generar mayor adiposidad abdominal. ${ }^{6,8}$ La menor percepción de estrés se asocia con mayor calidad de vida en la dimensión de salud mental del paciente con obesidad o sobrepeso. ${ }^{7}$

En este sentido, diversos estudios han descrito que el paciente con obesidad presenta mayor vulnerabilidad: es objeto de burlas sobre su peso, su autoimagen no es favorable, siente mayor dolor corporal, consumo comida de menor calidad, su actividad física es escasa y pasa mayor tiempo frente a la televisión. ${ }^{9,10}$

La forma como los pacientes con obesidad abordan las diversas situaciones estresantes se relaciona con su estilo de afrontamiento, el cual se ha descrito como un proceso en el que se hacen constantes esfuerzos cognitivos y conductuales para manejar el estrés psicológico generado por situaciones internas o externas percibidas como amenazantes o desbordantes; se considera una tendencia desarrollada a lo largo de la vida para utilizar un método que permita hacer frente a las situaciones estresantes. Generalmente se pueden reconocer dos grandes tipos de afrontamiento: dirigido a la acción y dirigido a la emoción. ${ }^{11,12}$

En un estudio que evaluó los estilos de afrontamiento de pacientes con obesidad mórbida se reportó que las mujeres mostraban mayor afrontamiento pasivo y evitativo, que se asoció con enojo, así como poca confianza y sin cercanía en las relaciones personales. ${ }^{12}$ En otro estudio se identificó que los pacientes con obesidad mórbida candidatos a cirugía bariátrica no afrontan eficazmente las situaciones problema debido a la estigmatización de la obesidad, la cual afecta su autoestima y autoconcepto, además de que posiblemente el afrontamiento evitativo se relacione con mayor porcentaje de depresión. ${ }^{13}$ De igual forma se ha informado que los pacientes que eligen la cirugía bariátrica como tratamiento presentaron un estilo de afrontamiento basado en las emociones y no en una dimensión activa, lo cual se puede asociar con mayor distrés y comportamientos mal adaptativos. ${ }^{12,14}$

Si bien la asociación entre estrés y obesidad, así como el tipo de afrontamiento hacia el estrés y su efecto en la calidad de vida en individuos con sobrepeso y obesidad han sido ampliamente estudiados, son escasas las investigaciones que evalúan estas variables en población candidata a cirugía bariátrica en México, lo cual deja un gran vacío en esta área de conocimiento debido a que la población latina es propensa al afrontamiento emocional, relacionado con problemas de alimentación, sobrepeso, obesidad ${ }^{15} y$ que puede afectar negativamente los resultados de la cirugía bariátrica. ${ }^{14,16}$

Debido a lo anterior, el presente análisis tuvo como objetivo identificar y describir la asociación entre la estrategia de afrontamiento al estrés, la percepción de calidad de vida y el nivel de IMC en candidatos a cirugía bariátrica.

\section{Método}

Estudio analítico observacional transversal. Se incluyeron pacientes mayores de 18 años candidatos a cirugía bariátrica de la clínica de obesidad del Hospital General "Dr. Manuel Gea González", con un IMC $\geq 40$ o de 35 si presentaban comorbilidades. Se excluyeron los candidatos a balón intragástrico y quienes estuvieran en proceso psicoterapéutico. Se eliminaron aquellos que no contestaron todos los cuestionarios.

Los participantes fueron reclutados en las sesiones de primer contacto en la clínica de obesidad y cirugía bariátrica del hospital, las cuales consistieron en 
pláticas grupales psicoeducativas de aproximadamente 30 minutos, en donde se les proporcionaba información general sobre los procedimientos para ingresar a la clínica. Posteriormente, tres psicólogas de la clínica obtuvieron por escrito el consentimiento para la participación en la investigación previa información al respecto, distribuyeron los cuestionarios y las instrucciones precisas para llenarlos y prestaron asistencia para solventar dudas; el tiempo promedio de este procedimiento fue de 45 minutos. Finalmente se revisaron los expedientes de los pacientes para obtener los datos sociodemográficos, peso, talla e IMC. La recopilación de datos se realizó entre agosto de 2015 y marzo de 2016.

Se utilizó el Cuestionario de Afrontamiento del Estrés ${ }^{17}$ en su versión validada en México. ${ }^{18}$ El instrumento evaluó siete formas de afrontamiento agrupadas en dos grandes factores:

\section{Afrontamiento emocional}

- Expresión emocional abierta: manifestaciones expresivas hacia otras personas de la reacción emocional negativa relacionada con el problema.

- Evitación: pensar o hacer otras cosas ignorando el problema.

- Autofocalización negativa: enfocarse a lo negativo de sí mismo como responsable del problema.

\section{Afrontamiento racional}

- Búsqueda de apoyo social.

- Religión.

- Focalización en solución de problemas: acción directa y racional para solucionar las situaciones problemáticas.

- Reevaluación positiva: crear un nuevo significado positivo a la situación problema.

Las respuestas se evaluaron en una escala tipo Likert con un rango de 0 a 4 . El instrumento se califica sumando los valores marcados en cada reactivo por cada subescala y se identifica como estilo de afrontamiento predominante el que presenta mayor puntuación. La escala ha reportado buenos niveles de confiabilidad en el afrontamiento emocional (alfa de Cronbach de 0.71) y en el afrontamiento racional (alfa de Cronbach de 0.78).

El Inventario de Calidad de Vida y Salud (InCaViSa) $)^{19}$ evalúa la calidad de vida en pacientes con cualquier padecimiento crónico o agudo y con sensibilidad a las intervenciones. Está compuesto por 53 reactivos de opción múltiple con escala tipo Likert constituida por opciones de respuesta de nunca a siempre, que evalúan 11 áreas de la vida, enfocándose en:

- Deterioro en calidad de vida: preocupaciones, aislamiento, percepción corporal, funciones cognitivas, actitud hacia el tratamiento, tiempo libre, vida cotidiana y dependencia médica.

- Calidad de vida: desempeño físico, familia, redes sociales y relación con el médico.

- Percepción de calidad de vida total.

Las puntuaciones de cada reactivo se obtienen por sumatoria directa, donde 0 es igual a nunca, 1 a casi nunca, 2 a pocas veces, 3 a frecuentemente, 4 a casi siempre y 5 a siempre. Las escalas desempeño físico, familia, redes sociales y relación con el médico se califican al revés. El instrumento muestra consistencia interna (los coeficientes alpha van de 0.6831 a 0.9337 ) y validez concurrente adecuadas.

El IMC ${ }^{20}$ es el marcador más utilizado para identificar obesidad y sobrepeso en población adulta; se obtiene mediante la división del peso en kilogramos entre el cuadrado de la talla en metros. De esta manera se calcula una puntuación que permite clasificar en obesidad grado I (30 a 34.9 puntos), obesidad grado II (35 a 39.9 puntos) y obesidad grado III (arriba de 40 puntos).

Se aplicó la prueba de Kolmogorov-Smirnov para identificar normalidad en la muestra; al no encontrarla se decidió utilizar el coeficiente de correlación de Spearman para evaluar la asociación y fuerza entre la estrategia de afrontamiento al estrés, el nivel de obesidad y la percepción de calidad de vida en pacientes candidatos a cirugía bariátrica. Se consideró estadísticamente significativo una $p \leq 0.05$. Para abordar el objetivo específico se realizó un análisis descriptivo, tomando en cuenta medidas de tendencia central y dispersión. Para el análisis se utilizó el programa estadístico SPSS versión 15.

El estudio fue aprobado por el Comité de Investigación y de Ética en Investigación del hospital en donde se realizó el estudio, con el número de registro 46-14-2014.

\section{Resultados}

Se evaluaron 127 pacientes, de los cuales se excluyeron 26 debido a que no cumplieron con los criterios de inclusión; la población quedó conformada por 101 pacientes, de los cuales $81.2 \%$ fue del sexo 
femenino y $18.8 \%$ del masculino; el rango de edad fue de 17 a 70 años y la media de 38.92 (desviación estándar $[\mathrm{DE}]=11.8$ años). Otros datos sociodemográficos se describen en la Tabla 1.

En cuanto a las estrategias de afrontamiento, las puntuaciones más altas están enfocadas a estrategias de afrontamiento racional: focalización a la solución de problemas (promedio 14.21, rango 4-24, DE 4.99), reevaluación positiva (promedio 13.37, rango 4-23, DE 4.00), búsqueda de apoyo social (promedio 11.38, rango 0-24, DE 6.41) y religión (promedio 5.90, rango 0-24, DE 5.54). En cuanto a las estrategias basadas en el afrontamiento emocional se obtuvieron los siguientes valores: expresión emocional abierta (promedio 9.26, rango 1-24, DE 5.54), evitación (promedio 9.18, rango 1-24, DE 4.32) y autofocalización negativa (promedio 7.94, rango 0-21, DE 4.26).

En cuanto al InCaViSa, la media obtenida indicó que los pacientes tuvieron una buena calidad de vida (promedio 111.50, DE 1.936). Respecto a las subescalas que permiten identificar la calidad de vida, se encontró nivel muy alto en el área familiar (promedio 3.78, rango 0-20, DE 3.939), nivel alto en el desempeño físico (promedio 10.06, rango 2-18, DE 4.190), alto en las redes sociales (promedio 7.55, rango 0-20, DE 5.108) y en la relación con el médico (promedio 10.29 , rango $0-20$, DE 4.394). Por otro lado, en las subescalas del instrumento que indican deterioro en calidad de vida se identificó nivel muy alto en aislamiento (promedio 5.55, rango 0-19, DE 4.471) y en la actitud ante el tratamiento (promedio 4.75, rango 0-20, DE 3.640); se encontraron niveles altos en las preocupaciones (promedio 9.54, rango 0-20, DE 4.896), funciones cognitivas (promedio 7.56, rango 0-18, DE 4.092), tiempo libre (promedio 9.52, rango 0-20, DE 5.409), percepción de la vida cotidiana (promedio 9.06, rango 0-20, DE 5.841) y dependencia médica (promedio 7.50 , rango 1-20, DE 4.358). Por último, la percepción corporal fue baja (promedio 13.70, rango 0-20, DE 4.747).

De las cuatro categorías que conforman el afrontamiento racional, tres tuvieron asociaciones negativas, y aunque débiles fueron estadísticamente significativas: focalización a la solución de problemas $\left(r_{s}=-0.2791\right.$, $p=0.005)$, reevaluación positiva $\left(r_{s}=-0.211, p=0.034\right)$ y búsqueda de apoyo social $\left(r_{s}=-0.249, p=0.012\right)$, con excepción de la religión, cuyo valor no fue estadísticamente significativo. En cuanto a las correlaciones obtenidas en el afrontamiento emocional, dos de las tres categorías presentaron asociaciones positivas,
Tabla 1. Caracterización demográfica de candidatos a cirugía bariátrica

\begin{tabular}{|c|c|c|c|c|c|c|}
\hline & Frecuencia & & Mínimo & Máximo & Media & DE \\
\hline Mujer & 82 & Edad & 17 & 70 & 38.92 & 11.81 \\
\hline Hombres & 19 & $\mathrm{Kg}$ & 62 & 220 & 113 & 29.05 \\
\hline \multirow[t]{2}{*}{ Total } & 101 & $\mathrm{~T}$ & 1.42 & 1.87 & 1.62 & 0.08 \\
\hline & & IMC & 26.48 & 77.19 & 42.41 & 8.92 \\
\hline
\end{tabular}

débiles pero estadísticamente significativas: expresión emocional abierta $\left(r_{s}=0.351, p=0.001\right)$ y autofocalización negativa $\left(r_{s}=0.461, p=0.001\right)$; en la evitación no se obtuvieron valores significativos (Tabla 2). Finalmente, se correlacionaron los siete tipos de afrontamiento con el IMC y no se obtuvieron asociaciones significativas (Tabla 3).

\section{Discusión}

El presente estudio tuvo como objetivo identificar la asociación entre la estrategia de afrontamiento al estrés, la percepción de calidad de vida y el nivel de IMC en pacientes candidatos a cirugía bariátrica. De igual forma se describieron los tipos y estrategias de afrontamiento al estrés más frecuentes en esta población. De acuerdo con los resultados, los pacientes de este estudio mostraron estrategias de afrontamiento enfocadas a la acción o la parte racional (focalización a la solución de problemas, reevaluación positiva y búsqueda de apoyo social), lo que llama la atención debido a que, de acuerdo con la literatura internacional, predomina el afrontamiento emocional en esta población. ${ }^{12-14}$ Una posible explicación es lo observado en la práctica clínica: al cuestionar a los pacientes respecto a su red de apoyo, la mayoría refiere contar con al menos un familiar que le proporciona apoyo significativo en momentos de dificultad, principalmente económica. Otra posible razón es que los participantes respondieron los instrumentos con deseabilidad social -indicaron lo que creían que los investigadores deseaban escuchar-, lo cual pudo generar la diferencia en los resultados. Existen investigaciones en las que se ha registrado la discrepancia entre la información de salud percibida por una población y los resultados de laboratorio. ${ }^{21}$

En cuanto a la calidad de vida, los datos muestran que los participantes perciben buena calidad de vida, particularmente en el área familiar, desempeño físico, redes de apoyo social y relación con su médico 
Tabla 2. Correlación entre calidad de vida, estrategias de afrontamiento al estrés e índice de masa corporal en candidatos a cirugía bariátrica

\begin{tabular}{|c|c|c|c|c|c|c|c|c|}
\hline & IMC & FSP & AFN & REP & EEA & EVT & BAS & RLG \\
\hline CaViSa & 0.145 & -0.279 & & & & & & \\
\hline & 0.148 & 0.005 & 0.001 & 0.034 & 0.001 & 0.818 & 0.012 & 0.563 \\
\hline \multicolumn{9}{|c|}{$\begin{array}{l}\text { Las correlaciones estadísticamente significativas se indican en negritas. IMC = índice } \\
\text { de masa corporal, FSP = focalización en solución de problemas, AFN = autofocalización } \\
\text { negativa, REP = reevaluación positiva, EEA = expresión emocional abierta, } \\
\text { EVT = evitación, BAS = búsqueda de apoyo social, RLG = religión, InCaViSa = Inventario } \\
\text { de Calidad de Vida y Salud. }\end{array}$} \\
\hline
\end{tabular}

Tabla 3. Correlación con el índice de masa corporal y las estrategias de afrontamiento al estrés en candidatos a cirugía bariátrica

\begin{tabular}{lccccccc}
\hline & FSP & AFN & REP & EEA & EVT & BAS & RLG \\
\hline IMC & -0.011 & 0.107 & 0.084 & 0.058 & 0.096 & 0.040 & -0.075 \\
$\mathrm{p}$ & 0.915 & 0.288 & 0.405 & 0.565 & 0.338 & 0.690 & 0.457
\end{tabular}

IMC = índice de masa corporal, FSP = focalización en solución de problemas, AFN = autofocalización negativa, $\mathrm{REP}=$ reevaluación positiva, $\mathrm{EEA}=$ expresión emocional abierta, EVT = evitación, BAS = búsqueda de apoyo social, $R L G=$ religión.

tratante, sin embargo, reconocen deterioro alto en aislamiento (sentimientos de soledad o separación de su grupo habitual a raíz de la obesidad), actitud ante los tratamientos médicos y preocupaciones asociadas con la obesidad. La percepción de alta calidad de vida contrasta con lo reportado en la literatura internacional, ya que diversos estudios mencionan que existe menor calidad de vida en pacientes con sobrepeso y obesidad, ${ }^{7}$ así como en los candidatos a cirugía bariátrica, y que las áreas más afectadas son movilidad, posibilidad de realizar actividades cotidianas, dolor, ansiedad y depresión. ${ }^{22}$

Diversos factores propios de la idiosincrasia latinoamericana ${ }^{23}$ podrían estar influyendo en la forma positiva y optimista como los pacientes perciben los eventos relacionados con su estado de salud, aun cuando estén padeciendo enfermedades críticas, con lo cual se explicaría las diferencias en la percepción de la calidad de vida. ${ }^{24}$ Además de lo descrito, es posible que los participantes respondieran los instrumentos del estudio con deseabilidad social. ${ }^{21}$

Respecto a las correlaciones se obtuvo que tres estilos de afrontamiento enfocados a la acción (focalización a la solución de problemas, reevaluación positiva y búsqueda de apoyo social) estuvieron asociados negativamente, si bien con una débil significación estadística, con el deterioro en la calidad de vida, es decir, a mayor focalización a la solución de problemas, reevaluación positiva y búsqueda de apoyo social, menor deterioro en la calidad de vida y viceversa. Los datos obtenidos no son raros, ya que diversos estudios han reportado que los estilos de afrontamiento enfocados a la acción suelen estar relacionados con conductas más adaptativas en situaciones de enfermedad e incluso conducir a la búsqueda de información sobre diversos padecimientos, entre ellos el cáncer. ${ }^{25}$

De igual manera, se obtuvieron correlaciones positivas, débilmente significativas, entre dos categorías de afrontamiento basado en la emoción (autofocalización negativa y expresión de la reacción emocional negativa relacionada con el problema) y el deterioro en la calidad de vida. Es decir, a mayor autofocalización negativa y expresión de la reacción emocional negativa relacionada con el problema, mayor deterioro en la percepción de calidad de vida. Estos datos son congruentes con los informados en la literatura internacional: los pacientes con obesidad y candidatos a cirugía bariátrica manifiestan un estilo de afrontamiento enfocado a lo emocional, por lo cual suelen presentar enojo, depresión, distrés, relaciones personales con poca confianza y comportamientos mal adaptativos, que finalmente merman su calidad de vida. ${ }^{12-14}$

Como se ha observado con anterioridad, ${ }^{26}$ en la población con obesidad no se obtuvieron relaciones significativas entre el IMC y los estilos de afrontamiento, los cuales al parecer únicamente tienen efectos mediadores en las emociones y comportamientos no saludables. ${ }^{9,10,12-14}$

Como limitación de la presente investigación se identificó la deseabilidad social con la que los pacientes respondieron a los cuestionario. ${ }^{21}$ En la práctica clínica, los pacientes candidatos a cirugía bariátrica manipulan la información debido a que desde el primer contacto se les indica que serán sometidos a un proceso de evaluación y preparación que determinará si son o no candidatos a cirugía. Este fenómeno obstaculiza la obtención de información verídica y dificulta la adecuada identificación y preparación de los pacientes para cirugía bariátrica. Posteriormente valdrá la pena evaluar este fenómeno para determinar el efecto que ha tenido en las investigaciones sobre esta población. También, la naturaleza transversal de este estudio impide analizar los estilos de afrontamiento empleados por los pacientes en diferentes momentos de su vida y en diferentes situaciones, información relevante tomando en cuenta que pueden cambiar dependiendo de la existencia de otras variables. De igual forma, no se realizaron análisis estadísticos más complejos que pudieran dar más información de las relaciones entre las variables para establecer causalidad. Por último, el presente estudio fue realizado 
solamente en un centro bariátrico de la Ciudad de México, lo cual no permite la generalización de los hallazgos a la totalidad de la población mexicana.

Se recomienda replicar este estudio de manera multicéntrica, incluyendo otras clínicas de cirugía bariátrica, empleando análisis estadísticos más complejos y líneas de investigación que estudien la deseabilidad social en esta población.

\section{Bibliografía}

1. World Health Organization [sitio web]. Obesity and overweight. Disponible en: http://www.who.int/mediacentre/factsheets/fs311/en.

2. WHO Expert Consultation. Appropriate body-mass index for Asian populations and its implications for policy and intervention strategies. Lancet. 2004;363(9403):157-163.

3. Rahman MM, Akter S, Jung J, Rahman MS, Sultana P. Trend, projection, and appropriate body mass index cut-off point for diabetes and hypertension in Bangladesh. Diabetes Res Clin Pract. 2017;126:43-53.

4. Organización para la Cooperación y el Desarrollo Económicos. Obesity update 2012. Francia: Organización para la Cooperación y el Desarrollo Económicos; 2012.

5. Alegre-Díaz J, Herrington W, López-Cervantes M, Gnatiuc L, Ramírez R, Hill M, et al. Diabetes and cause-specific mortality in Mexico City. N Engl J Med. 2016;375(20):1961-1971.

6. Wardle J, Chida J, Gibson EL, Whitaker KL, Steptoe A. Stress and adiposity: a meta-analysis of longitudinal studies. Obesity (Silver Spring). $2011 ; 19(4): 771-778$.

7. Wang J, Sereika SM, Styn MA, Burke LE. Factors associated with health-related quality of life among overweight or obese adult. J Clin Nurs. 2013;22(15-16):2172-2182.

8. Adam T, Epel ES. Stress, eating and the reward system. Physiol Behav 2007:91(4):449-458.

9. Buttitta M, Iliescu C, Rousseau A, Guerrien A. Quality of life in overweight and obese children and adolescents: a literature review. Qual Life Res. 2014;23(4):1117-1139

10. Mouchacca J, Abbott GR, Ball K. Associations between psychological stress, eating, physical activity, sedentary behaviors and body weight among women: a longitudinal study. BMC Public Health. 2013:13:838.

11. Lazarus RS, Folkman S. Stress, appraisal, and coping. EE. UU.: Springer; 1984.
12. Hörchner R, Tuinebreijer WE, Kelder H, Van-Urk E. Coping behavior and loneliness among obese patients. Obes Surg. 2002;12(6):864-868.

13. Fettich KC, Chen EY. Coping and obesity stigma affects depressed mood in African-American and white candidates for bariatric surgery. Obesity (Silver Spring). 2012; 20(5):1118-1121.

14. Rydén A, Karlsson J, Persson LO, Sjöström L, Taft C, Sullivan M. Obesity-related coping and distress and relationship to treatment preference. Br J Clin Psychol. 2001;40(Pt 2):177-188.

15. Reyes-Rodríguez ML, Gulisano M, Silva Y, Pivarunas B, Luna-Reyes KL, Bulik CM. "Las penas con pan duelen menos": the role of food and culture in Latinas with disordered eating behaviors. Appetite. 2016;100:102-109.

16. Rydén A, Karlsson J, Sullivan M, Torgerson JS, Taft C. Coping and distress: what happens after intervention? A 2-year follow-up from the Swedish Obese Subjects (SOS) study. Psychosom Med. 2003;65(3): 435-442.

17. Sandín B, Chorot P. Cuestionario de Aprontamiento del Estrés (CAE): desarrollo y validación preliminar. Revista de Psicopatología y Psicología Clínica. 2003;6(1):39-54

18. González M, Landero R. Cuestionario de Afrontamiento del Estrés (CAE): validación en una muestra mexicana. Revista de Psicopatología y Psicología Clínica. 2007;12(2):189-198.

19. Riveros-Rosas A, Sánchez-Sosa JJ, Del Águila M. Inventario de Calidad de Vida y Salud (InCaViSa). México: El Manual Moderno; 2009.

20. Regional office for Europe. Body Mass Index - BMI. World Health Organization; 2018. Disponible en: http://www.euro.who.int/en/health-topics/ disease-prevention/nutrition/a-healthy-lifestyle/body-mass-index-bmi

21. Mossavar-Rahmani Y, Tinker LF, Huang Y, Neuhouser ML, McCann SE, Seguin RA, et al. Factors relating to eating style, social desirability, body image and eating meals at home increase the precision of calibration equations correcting self-report measures of diet using recovery biomarkers: findings from the Women's Health Initiative. Nutr J. 2013;12:63.

22. Twells LK, Driscoll S, Gregory DM, Lester K, Fardy JM, Pace D. Morbidity and health-related quality of life of patients accessing laparoscopic sleeve gastrectomy: a single-centre cross-sectional study in one province of Canada. BMC Obes. 2017;4:40.

23. Trapp SK, Perrin PB, Aggarwal R, Peralta SV, Stolfi ME, Morelli E, et al. Personal strengths and health related quality of life in dementia caregivers from Latin America. Behav Neurol. 2015;2015:507196.

24. Lavielle-Sotomayor P, Rozen-Fuller E, Bustamante-Rojano J, Martínez-Murillo C. Optimismo, cohesión familiar y tratamiento como predictores de la calidad de vida en padecimientos oncohematológicos. Rev Med Inst Mex Seguro Soc. 2017;55(4):456-463.

25. Saita E, Acquati C, Kayser K. Coping with early stage breast cancer: examining the influence of personality traits and interpersonal closeness. Front Psychol. 2015;6:88.

26. Kaymaz N, Düzçeker Y, Uzun ME, Aylanç H, Baștürk M, Yıldırım S. Influence of body mass index on mindfulness awareness and coping methods for stress in adolescents. Int J Adolesc Med Health. 2016;30(3):pii. 